\title{
The Salesperson with a Speech Impediment: An Objective Research and Analysis on the Importance of Clarity, Structure and Logic of Arguments
}

\author{
Martin D Chekuri CF. \\ APMP®, MBA Strategic Management, MSc Psychology, \\ Vice Chairman APMP Hyderabad Chapter | Proposal Writer, Shipley Associates
}

Author Note

This research paper and its presentation was funded by Shipley Associates and was prepared for presentation at the International Conference on Social Sciences XVII, Merced Campus University of Murcia, Spain

\begin{abstract}
There is no sales document as crucial to winning business deals as the business proposal document. We noticed that business proposals try their best to be persuasive, and yet, they do not have the impact they hope to create. In order to understand why we conducted detailed research and found out that customers simply refuse to read some proposals. There are three reasons for this: Logic, Clarity, and Aesthetics. Our research showed that the lack of logic or the presence of logical fallacies account was the reason most often quoted by customers $63 \%$ of the times) for not wanting to read the business proposal, followed by clarity, at $29 \%$ and aesthetics at $9 \%$. To write this research paper, we've focused primarily on understanding logical fallacies, categorizing them and identifying which of them are the most identifiable and which of them are most frequent. We also recommend a systematic way to avoid logical fallacies in proposals.
\end{abstract}

Keywords: Logic, Logical Fallacy, Clarity, Aesthetics, Business Proposals

\section{Introduction}

By definition, a speech impediment is a hindrance to clear communication. It acts as an encumbrance upon the speaker and a restraint upon the listener. As a result, one will almost never find a salesperson with a speech impediment.

A business proposal does the same things that a salesperson must do. However, business proposals have challenges that salespersons do not. A salesperson can have a conversation with a customer. In the process, they can answer questions, provide clarifications and understand when the customer is bored, disinterested or has a deep aversion towards what they are listening to. Almost instantly, the salesperson can change the topic or make subtle changes in the way they converse, crack a joke or say something to regain the customer's trust or reduce the customer's aversion.

Usually, salespersons are among the most sharply dressed employees of an organization. By the way they appear, they hope to make a good first impression. They aim to win business for their respective organizations. They try their best to make a sound value proposition to the customer and become the most favored salesperson among the salespersons who are competing for the attention of the customer. They aim to sound empathetic, logical and clear.

The lack of these attributes create an unnecessary speech impediment and cause customers to show aversion towards the information they receive.

A business proposal is much like a salesperson. Yet, it does not see the customer face to face in real time. It fails to understand their mood and then communicate. As a result, it sounds robotic to the reader.

To understand why proposal evaluators get turned off while reading a proposal, we asked them what emotions they go through and what reasons they cite for rejecting a business proposal. Our assessments led us to understand that business proposals are like salespersons with speech impediments. 
But often, a business proposal has at least one significant speech impediment. It fails to be logical and clear. As a result, it invites the dislike of the evaluator who is reading it.

In our research in the past, we assumed that the lack of clarity was the reason that deterred most customers from reading a business proposal. While there is significant evidence to substantiate the negative impact of complex jargon and convoluted sentences on readers, our recent studies showed us a greater, more significant reason for customers disposing business proposals without reading them. Logic, or the lack of it was the greatest contributor to customer's instant aversion towards a business proposal. The lack of logic combined with the lack of clarity often was the perfect recipe for instant proposal aversion.

\section{Proposal Aversion}

Proposal evaluators consider their job to be extremely tedious, time-consuming, boring and hectic. They do not possess the time nor the patience to read business proposals. Unfortunately for them, business proposals tend to be lengthy. While some proposals are just about 20 pages long, most business proposals are about 100 pages long. In some cases, as often seen in the case of federal bids in the United States, proposals can be over 1000 pages long, and may require the pooling of multiple evaluators to read through proposals and award a bid. This was the primary cause of the reduced motivation to read the proposal; A simple lack of desire because of the tediousness of the work involved. However, we understood from proposal evaluators that there were other factors that in fact caused what we termed, "proposal aversion."

We define proposal aversion as "the state of mind a proposal evaluator attains when they dislike a proposal and make a conscious or subconscious decision to not award the bid to the organization that authored the proposal- after reading the proposal (in part, or in whole)."

We asked forty-two (42) senior managers from across the globe who have had experience in evaluating business proposals on what caused proposal aversion, and at what stage they arrived at the aversion.

Based on their input, we found that they developed an aversion towards a business proposal if it had a fallacious claim or was backed up by poor logic. This was recorded $63 \%$ of the times. The lack of clarity was another culprit, which we recorded $29 \%$ of the times. Interestingly, although we assumed this would be a significantly important factor, the aesthetics, coverpages, graphics and graphical descriptions of a proposal seemed to matter to only $8 \%$ of proposal evaluators.

Figure 1: Contributors to proposal aversion

\section{What contributes the most to proposal aversion?}

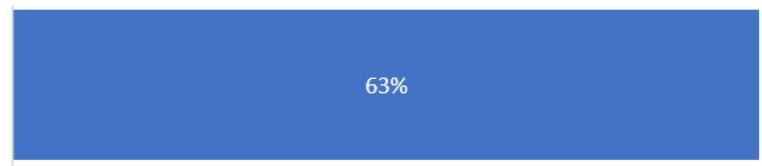

Convoluted statements, jargon

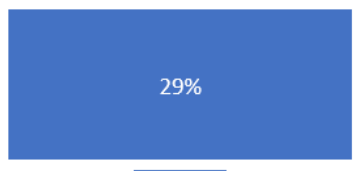

Poor aesthetics, graphics or design

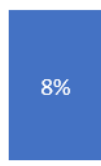

Proposal aversion was most often noticed after the evaluator read a significant portion of the proposal. $24 \%$ of all proposal evaluators claimed that a poorly written executive summary (chapter one) of the proposal was a contributor to proposal aversion. Most of the evaluators (64\%) claimed that they faced aversion after reading about $20-40$ pages. The rest claimed that they reached aversion after they finished reading the proposal. 
Figure 2: Proposal Aversion and its Development

\section{When do you usually develop proposal aversion?}

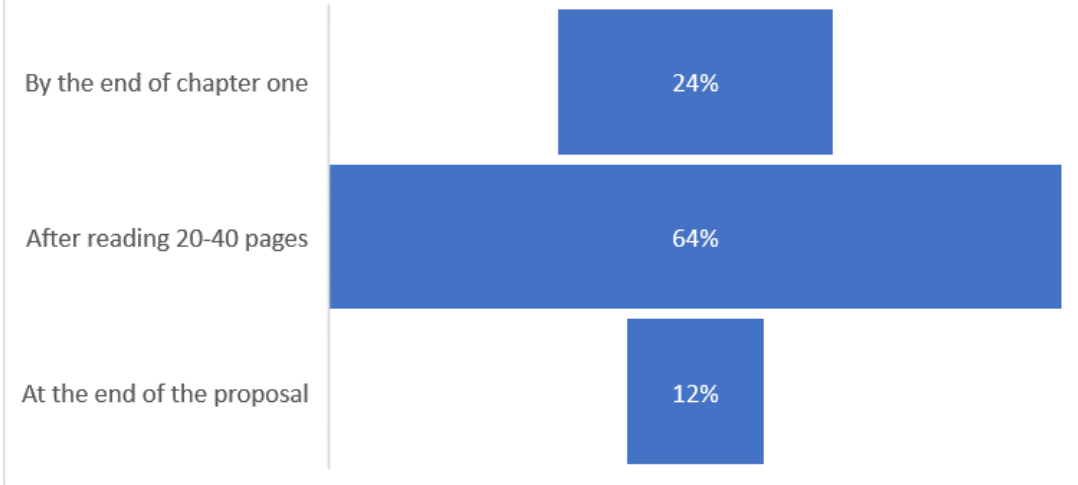

\section{Logical Fallacies in the Salesman's Argument}

A logical fallacy is the use of the wrong reason to construct an argument. The word "argument" has its origins in the word "arguere" from Latin, which means, "to make clear, prove or accuse." One of the most respected definitions of the word "argument" was given by Van Eemeren.

"Argumentation is a verbal and social activity of reason aimed at increasing (or decreasing) the acceptability of a controversial standpoint for the listener or the reader, by putting forward a constellation of propositions intended to justify (or refute) the standpoint before a rational judge" (Eemeren, 1999)

As suggested by Van Eemeren, at the heart of an argument lies the salesperson's opinion, inference or assertion that causes them to gain a "reason." This reason is put into words and stated to the listener or reader. Then, the reason is backed up by more propositions to solidify the argument.

\section{What causes a logical fallacy?}

Any logical fallacy begins with the salesperson desiring to make a point through reasoning. The reasoning comes from inferences. (Walton, Fundamentals of Critical Argumentation, 2006) Sometimes, the reasoning does not adequately consider the various external factors that affect the argument. And hence, the argument finds itself to be fallacious. Sometimes fallacious logic is rooted in cognitive biases or partially viewed data (Schwarz \& Asterhan, 2008). Some other times, it is rooted in a completely unplanned interpretation of social information (Kahneman, 2011). Sometimes, it could just be because of an emotional appeal on the salesperson's mindset or the customer's mindset.

And yet at other times, it is just because the salesperson "thinks" so. 'Thinking' and 'reasoning' are completely different, with 'thinking' being sometimes, an involuntary process, but 'reasoning' being a voluntary, conscious effort. (Schwarz \& Asterhan, 2008)

As suggested above, there are many ways to arrive at a logically fallacious argument. One interesting point we may note here is that the correct inferences do not necessarily point to correct reasoning or correct arguments. Correct inferences point to correct reasoning and correct arguments only when they are channeled through a deliberate, conscious and verified process of unbiased thinking and reflection. (Moshman, 1995)

During our research, based on our conversations with customers, we identified many logical fallacies. While the subject of rhetoric and debate teach over 300 logical fallacies, (Bennett, 2013) we will restrict this study to the 10 most commonly found logical fallacies. 


\section{Ten Common Logical Fallacies and Their Examples}

Slippery Slope: If A happens, then B will happen.

The Slippery Slope Fallacy is a common occurrence in proposals, where proposal writers and contributors assume from prior experience with customers. Statements such as "If your servers are slow, your business will slow down too." While the statement is accurate, it is still an assumption. Hence, a better alternative would be "If your servers are slow, it may slow down your business too." Using the word "may" has a considerable impact on reducing the frequency of occurrence of the Slippery Slope logical fallacy (Lode, 1999)

Hasty Generalization: A \& B have an attribute "X," and hence, $C$ will have it too.

Hasty Generalization is much akin to Slippery Slope. There are two kinds of. logical fallacies under the umbrella term "Hasty Generalization." The first, 'Universal Generalization', is a fallacy where the argument applies a particular instance to a universal condition. The second, namely 'Secundum Quid' does the same thing as Universal Generalization, and also overlooks the qualifications of the premise. (Walton, Rethinking the Fallacy of Hasty Generalization, 1999)

Both these are common sightings in business proposals. The writer usually relies on previous experience and makes a predictive assertion without careful reasoning. The Hasty Generalization fallacy finds its place very often in business proposals where proposal writers make assertions such as "Your competitors, A \& B rely on our solution to meet their market needs. Our solution will certainly help you meet your market needs as well."

Post Hoc Ergo Propter Hoc: B happened after A. Therefore, B happened because of A

The Greeks realized this fallacy millennia ago. Because one event follows another event, it does not mean that the first event caused the second event. (Grouse, 2016) This is one of the most noticeable forms of logical fallacies. Customers understand consequential occurrences in their environments better than we do. Hence, business proposal writers must be aware that just because a customer's data shows consequential occurrences, there is no guarantee that it is because one event led to another. A statement such as "The data shows that the customer footfall is low on Monday because the day prior was Sunday" may seem like an adequate analysis. However, customers may have identified other reasons for this that the proposal writer is not aware of. In scenarios where an assumption as this is made, it is best to check before making an assertion.

Cum Hoc Ergo Propter Hoc: Correlation is not equal to causation.

This fallacy is very similar to the previously stated logical fallacy, Post Hoc Ergo Propter Hoc. However, it is slightly different. The latter suggestions causation because of the chronology of events. The former does not. (Eabrasu, 2015)

Cum Hoc Ergo Propter Hoc is the easiest logical fallacy to identify for customers who have spent sufficient time pondering over their own data.

Figure 3: Spurious Correlation between drowning and Nicolas Cage films: Why Correlation is not Causation (Vigen, 2015) 


\section{Number of people who drowned by falling into a pool \\ correlates with}

\section{Films Nicolas Cage appeared in}

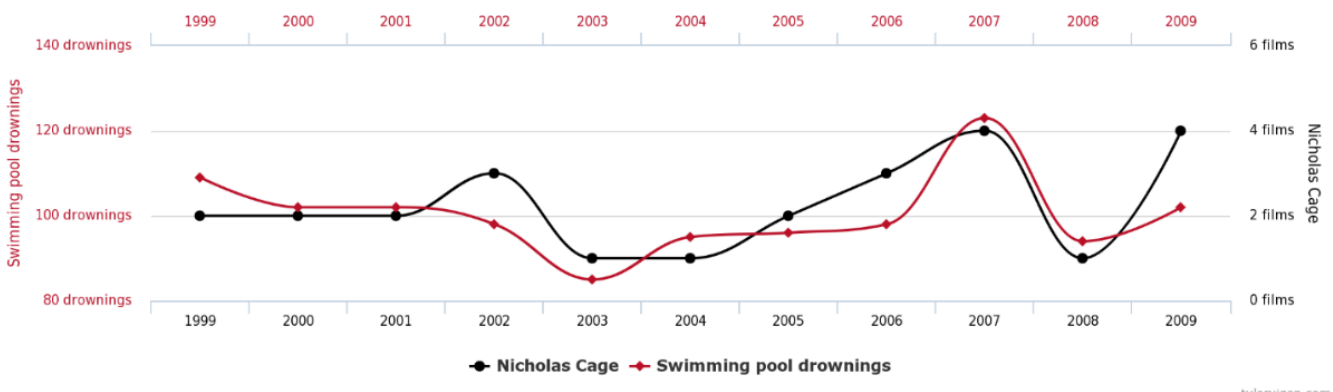

Eager business proposal writers make the mistake that data points put together suggest a certain pattern, and Therefore, correlate to support an argument. While correlation can imply causation, in some scenarios, it simply does not

False Dilemma/ Either Or Fallacy: If not $A$, then $B$. If not $B$, then $A$

The False Dilemma Fallacy is type of fallacy where an argument is based on two extremes; where there is infact, an alternate option. This is a fallacy not just common in business proposals but also a common fallacy in making a conclusion from corporate data analyses. The False Dilemma/ Either-Or Fallacy does not leave space for a middle ground. (Hanks, 2019)

In business proposals, they are commonplace through statements like "We understand your problem. It is likely caused by one of two factors, A or B." Although the statement may at first glance seem to be true, there is a likelihood that in the environment of the customer, it is caused by neither $\mathrm{A}$ nor $\mathrm{B}$, or both. If an assertion such as this must be made, it is best to check with the customer or state the assumption in the next line.

\section{Ad Populum: Everyone is doing it}

Ad Populum or the "Bandwagon" argument is a common logical fallacy. It is common in rhetoric, business circles and in proposals. Statements such as " $80 \%$ of our customers want to use our product again. We are sure you will use our product more than once too" are common occurrences. While the first statement is a good argument in itself to persuade someone to purchase the product, the second statement is a logical fallacy. In the European Union especially, business proposals and statements of work (SOWs) are taken very seriously. If a claim is made and it does not eventually pan out, the customer has the right to sue the vendor for the claim that had been made.

\section{Strawman Fallacy}

The strawman fallacy misrepresents the competitors' argument and tries to gain leverage from the same.

A common business proposal strategy is what is termed as "ghosting." The ghosting strategy aims to take down a possible competitor strategy. This is done by over-emphasizing competitor weaknesses or underplaying the importance of their strengths. While the tactic in itself is not ethically wrong to use, customers have identified it as a logical fallacy. Overemphasizing competitor weaknesses without enough emphasis on self-strength or vice versa often is viewed as ghosting poorly done. In scenarios as this, it is considered to be a strawman fallacy.

\section{Argument by Gibberish and Ambiguity}

While we have found that the lack of clarity is a major contributor to proposal aversion, in some cases, customers also viewed it as a logical fallacy. Argument by gibberish attempts to confuse customers so that they do not thoroughly understand what is being stated. Arguments by gibberish are often found in the Terms and Conditions chapter of a proposal. They are also found in some executive summaries where there is too much emphasis put on the features of the product/ solution rather than on the benefits of the same. 


\section{The Texas Sharpshooter Fallacy}

The name "Texas Sharpshooter Fallacy" comes from a funny anecdote about a certain Texan who fired gunshots at a barn, and then painted a target where most of his shots clustered, and then made the outlandish claim to be a sharpshooter.

A lot of times, selective data analysis is used by proposal writers to make a claim. The error of judgement is a logical fallacy and is easy to notice when the entire data sample is taken into consideration.

\section{Appeal to Authority}

The Appeal to Authority logical fallacy is often found in the executive summary of business proposals that aim to be persuasive. In a bid to make their arguments strong, proposal writers make an effort to find data from popular sources to aid their claims.

In our interviews with proposal evaluators, we've understood that the appeal to authority is only understood as a logical fallacy if subsequent points are not effective reasons for the argument.

\section{Data Analyses of Logical Fallacies and Their Impact in Business Proposals}

\section{The Most Frequent Logical Fallacies}

We studied and manually assessed 50 business proposals carefully over a span of a year. We identified that the most frequent logical fallacies were not the same as the most identified logical fallacies in the data sample that we considered. We assessed business proposals from multiple organizations and arrived at the following analysis.

Table 1

The Most Recurrent Logical Fallacies

Logical Fallacies: Observed Cumulative Frequency in 50 business proposals

\begin{tabular}{lll} 
Logical Fallacy & Frequency & Percentage \\
\hline Slippery Slope & 104 & $18 \%$ \\
Appeal to authority & 102 & $17 \%$ \\
Argument by Gibberish and Ambiguity & 95 & $16 \%$ \\
Strawman fallacy & 76 & $13 \%$ \\
Hasty Generalization & 64 & $11 \%$ \\
The Texas Sharpshooter & 52 & $9 \%$ \\
False Dilemma / Either-Or Fallacy & 40 & $7 \%$ \\
Ad Populum & 28 & $5 \%$ \\
Post Hoc Ergo Propter Hoc & 16 & $3 \%$ \\
Cum Hoc Ergo Propter Hoc & 12 & $2 \%$
\end{tabular}

\section{The Most Noticeable Logical Fallacies}

Although the above study gave us tremendous insight, we wanted to see if the most recurrent fallacies were also the most identified ones.

In our interviews with proposal evaluators, we asked: "Of all the logical fallacies, which are the most frequently observed fallacies?" We asked evaluators to pick three logical fallacies out of ten. Based on the data we gathered, we were informed that the following logical fallacies played the most significant role in deterring the interest of the evaluator. 
Table 2

The Logical Fallacies that Proposal Evaluators Identify and are Averse to

$\begin{array}{lllll}\text { Proposal Aversion Creating Logical Fallacies } & \text { 1st Place } & \text { 2nd place } & \text { 3rd place } & \text { Total } \\ \text { Hasty generalization } & 10 & 7 & 3 & 20 \\ \text { The Texas Sharpshooter } & 9 & 3 & 5 & 17 \\ \text { Argument by Gibberish and Ambiguity } & 7 & 4 & 4 & 15 \\ \text { Cum Hoc Ergo Propter Hoc } & 5 & 3 & 6 & 14 \\ \text { False Dilemma/ Either-Or Fallacy } & 2 & 7 & 3 & 12 \\ \text { Strawman fallacy } & 1 & 6 & 5 & 12 \\ \text { Appeal to authority } & 3 & 2 & 6 & 11 \\ \text { Post Hoc Ergo Propter Hoc } & 1 & 4 & 4 & 9 \\ \text { Ad Populum } & 2 & 3 & 4 & 9 \\ \text { Slippery Slope } & 2 & 3 & 2 & 7\end{array}$

\section{The Impact of Logical Fallacies}

While interviewing our 42 proposal evaluators, we asked them if proposal aversion is instantly caused by a logical fallacy, or if there is an intermediate emotion. In response to our query, $74 \%$ of the evaluators explained that logical fallacies cause a "suspicion" or a "breach of trust." Another $20 \%$ of them said that logical fallacies cause "doubts that hinder an unbiased viewing of the rest of the proposal." Only $6 \%$ of the proposal evaluators said that logical fallacies instantly caused "proposal aversion" without an intermediate emotion.

Figure 4: Intermediate Emotions before Proposal Aversion

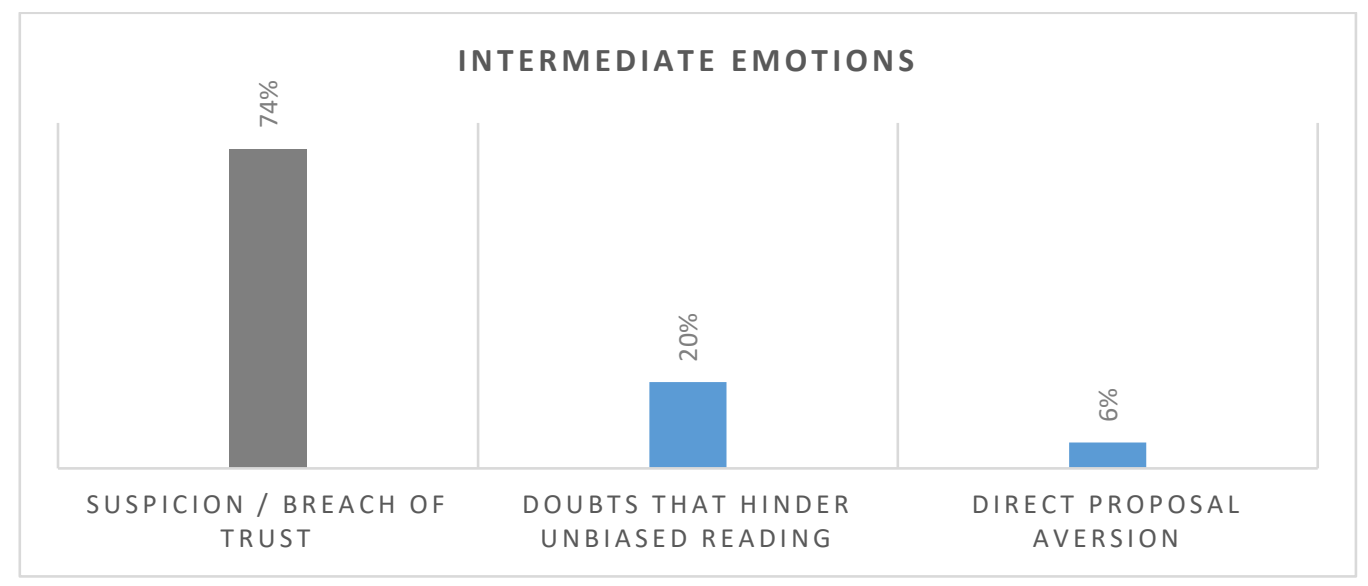

\section{Strategies to Make an Airtight Argument}

\section{The Structure of An Argument}

While there is no singular way to perfectly tackle and avoid all forms of logical fallacies in business proposals, we recommend following the following principles to reduce the frequency of their occurrence.

\section{Precision}

The first and most important way to articulate a sound argument is to start with the conclusion. What are you trying to prove in the first place? What do you want your reader to conclude after reading what you wrote? Asking yourself these questions 
over and over again helps you stay concise and crisp and not wander into irrelevant topics or make statements that would work against you.

Finally, each reason must be supported by a significant piece of evidence, the lack of which causes the reason cited to be unsubstantiated. Hence, the writer must play with multiple reasons, and remove reasons that are not relevant, and come up with curated, re-written reasons that are specific and relevant to those who are reading what they have been presented.

\section{Pyramids}

To reduce logical fallacies in your document, use the Minto Pyramid Principle in articulating your message. Using the pyramid principle will help you craft a crisp document that eliminates unnecessary content and helps you stay focused on your key message. Also look for content that does not add value to the reader, or which does not make sense in the pyramid.

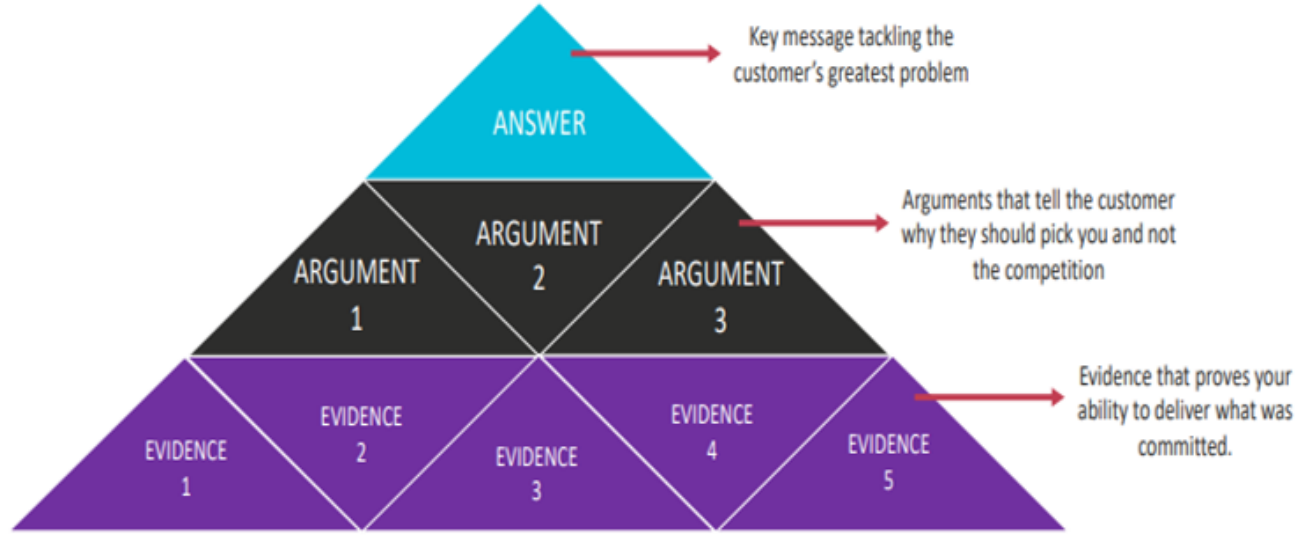

Figure 5: The Minto Pyramid Principle: Using this technique, proposal writers can ensure that proposals are focused on the key message- instead of confusing the reader (and the writer) (Minto, 1978)

\section{Paragraphs}

Another systematic way to avoid logical fallacies is by using Scott Lovell's Structure for Arguments by Systematic Outlining (Lovell, 2009) as follows:

Paragraph 1: Introduction and Thesis

Paragraph 2: Statement of Fact and Definitions

Paragraph 3: Proof 1

Paragraph 4: Proof 2

Paragraph 5: Proof 3

Paragraph 6: Counterpoints

Paragraph 7: Systematic refutation of counterpoints

Paragraph 8: Conclusion

An alternative, if the above recommendation seems rather long for a proposal; is to combine paragraphs 3,4 and 5 can be combined into a single paragraph. Paragraph 6 and 7 can also be combined into a single paragraph to reduce the amount of content that goes to make a strong, air-tight argument.

\section{The Strength of An Argument}

The strength of a man is dependent on his skeleton. And then on the muscle that lies on top of it. And then on the strength of the cells and tissues that have developed his body. 
Quite similarly, the strength of an argument is dependent on the things that make it. The reasons it provides and the evidence it lays out.

Step 2 is to find reasons for your reader to agree with you. Hence, the number of reasons you give them is not as important as the weight the reasons have on the mind of the reader. You cannot give an automobile company the reasons which are relevant to a pen factory. Even if the reason is relevant, it must be modified so that the reader finds it relevant to his problem. No two reasons must be similar. If they are similar, it is best to combine them under one heading. Too many reasons are not good too, for people have trouble remembering more than three at a time.

\section{Sincerity}

One can often notice that the strength of the argument is largely dependent on the conviction of the writer. If the writer is asked to showcase the strength of a dinosaur when he is asked to write on chickens, it is hardly possible. This causes the writer to start coming up with content that he himself does not agree with. Which in turn becomes an unnecessary set of words that remove the steam out of the argument and make it sound feeble. One must always remember the adage, "Empty vessels make much noise."

\section{Sequence}

In a proposal, the sequence of arguments is vital to clarity. A set of arguments that are jumbled cause confusion. Anything that is confusing causes a significant amount of fog in the mind of the reader. Hence, it is important for statements and paragraphs to have a logical sequence to them. The ideal argument unfolds like a handkerchief. It is not like a messy roll of cloth stuffed into the crevice of a messy suitcase. Instead, it is sequentially arranged. The conclusion of your argument is articulated in the beginning, and the message, the reasoning, and the evidence that support the argument unfold systematically.

\section{Specifics}

Numbers are impactful when you state evidence to prove a point you are trying to make. To persuade someone that you are good at getting results, you should also subtly make the other person question how many times they've missed getting the said results. This is why numbers are critical to making an argument more persuasive. More so, are infographics that cite numbers. You can read more about the representation of numbers in our next chapter on information design.

\section{The Substantiation of An Argument}

\section{Context}

Any argument, no matter how precise, well laid out, sincere or specific, will not resonate with the customer if it is not contextually accurate. If the content and the proposal strategy that went into it were designed for a retail company, it would seldom work for an automobile company. Rewrite content keeping the customer's industry in mind. Ensure that evidence quoted is also relevant to the customer and not something that is completely disconnected.

A surprisingly common logical fallacy is the red herring. Red herrings involve bringing up irrelevant information to argue your case or to debunk the evidence of others. It's important to avoid red herrings because they make your argument look elementary. Even if you are arguing a strong point and are right to do so, including red herrings in your document creates glaring holes in your reasoning and turns readers away from your positions.

To avoid using red herrings, make sure that your argument is always framed within the context of the argument and not your personal feelings on the issue.

If you notice a red herring in your document, take the time to re-examine your evidence for the issue so that you can more clearly define it for readers. As red herrings are often quite obvious and distracting, removing them from your document is crucial to having a polished and persuasive final product.

\section{Corroboration}

Always have more than one point of evidence. Anything more than three is overkill, anything less is shabby. One piece of evidence seldom convinces a reader to generalize. Multiple pieces of evidence support their logic. Hence, the more, the 
better. But don't let that get in the way of making your business document concise. If necessary, add a link where interested readers can click to read more about other businesses you've helped.

\section{Circumventing}

The word "circumvent" means, "find a way around." Being prepared for opinions that counter your argument is an excellent practice. This is a better strategy than hoping that the reader does not find a counterpoint. Ensure that your writing at least addresses the obvious counterpoints that come against your arguments. Make it a point to call out the logical fallacies in the counterpoints against your argument.

\section{Conclusion}

Once you have ensured that all counterpoints against your argument are addressed, tighten any loose ends, and restate your initial thesis. Usually, writers place a lot of effort to hook their readers at the beginning, but the steam goes off towards the end. Always aim to leave your reader with something to think about at the end. End with a question for the reader to think about or a call to action. Ensure that you have sufficiently edited your content once you are done with the writeup.

\section{Conclusion}

Implement the Barbara Minto Principle to develop a proposal that eliminates insignificant content.

Implement Lovell's Structure of Arguments to develop logically airtight content while developing a persuasive document

Keeping your content concise and tight reduces the likelihood of logical fallacies.

Avoid having multiple contributors adding text to the document. Multiple contributors inevitably cause conflicting ideas to creep into the proposal; thereby creating logical fallacies

If multiple contributors must add content, ensure that content is carefully evaluated to assess and find logical fallacies.

Ensure that the person responsible for reviewing the document knows what logical fallacies are and is trained to find them.

You can avoid the logical fallacies created by gibberish and ambiguity by paying attention to readability statistics and using them extensively to evaluate the quality and readability of your content. Our research on the subject of ambiguity in proposals showed that complexities that arise from writing techniques actually cause customers to develop a distaste for the proposal. In one study, thirty people in a local Starbucks were given two documents each. One document was compiled with a lot of jargon and long sentences. The other was simple and easy to understand. Both were in effect- trying to share the same message. After the subjects read the two documents, they were given a list of character traits. The list of character traits had fifteen positive ("good") and fifteen negative ("bad") traits. Readers were asked to associate each document with a list of traits. The study found that $100 \%$ of the readers associated the document with jargon and complicated sentences with "obnoxiousness, rudeness, stubbornness, and unreliability." Not a single positive trait was attributed to the complex document. (Fugere, Hardaway, \& Warshawsky, 2005)
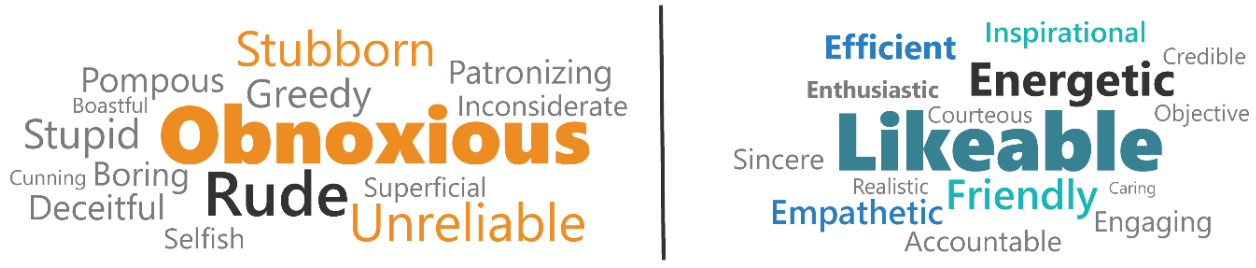

Figure 6: How Readability Influences Likeability: Fugere's research shows us that the readers despise content that is hard to read. Complicated writing styles were found to be obnoxious and unreliable- while documents that were easy reading was found to be likable and empathetic. (Fugere, Hardaway, \& Warshawsky, 2005)

Data and statistics are often wrongly used as an end-all argument. However, data is complicated and can be subjected to all forms of interpretations While using data and statistics, challenge "cause" and "effect." 
Schedule "Editing" while planning your proposal. Ensure that the tasks are clear, and the editor knows what logical fallacies are and how to find them.

Use a systematic strategy to content development to reduce the likelihood of logical fallacies. You may choose to use the strategic plan in the next page to aid your writing plan.

\section{Summary: Strategies to Make}

Figure 7: The 3S Model of Developing Airtight Arguments

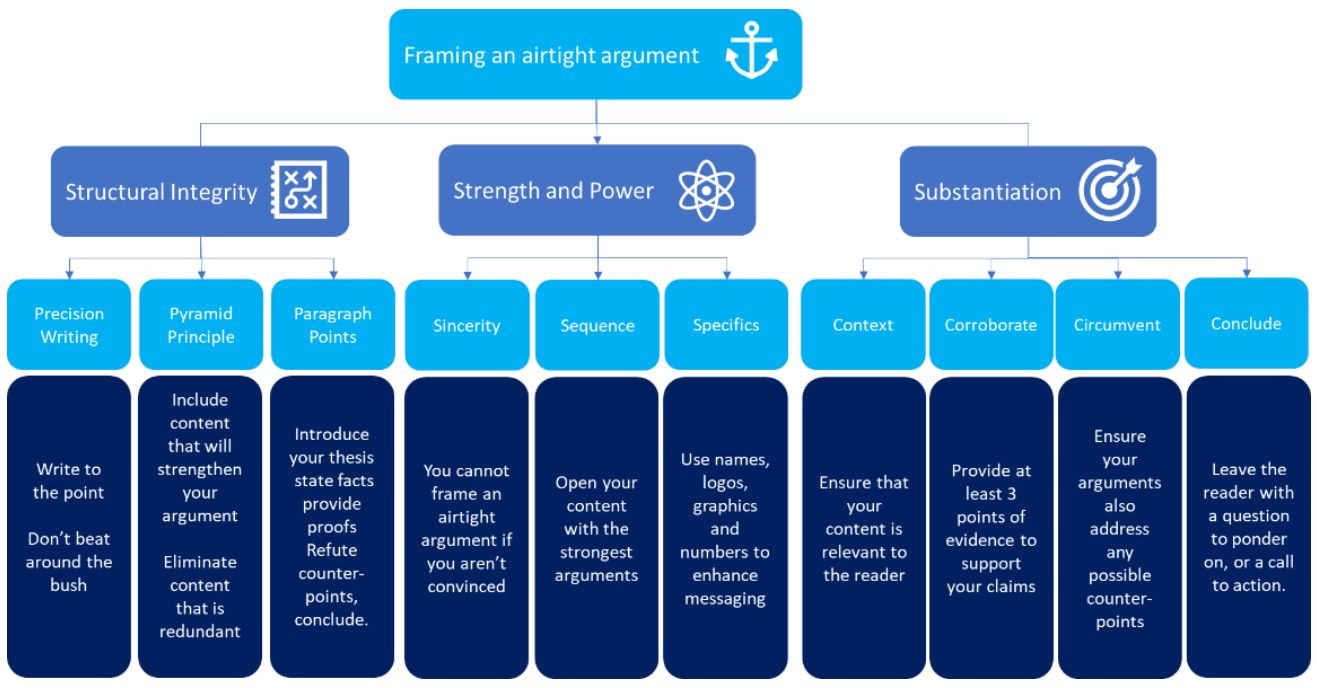

\section{An Airtight Argument}

\section{References}

[1] Bennett, B. (2013). Logically Falacious: The Ultimate Collection of Over 300 Logical Fallacies. Amazon E Books.

[2] Eabrasu, M. (2015, July 15). Post Hoc Ergo Propter Hoc: Methodological Limits of Performance Oriented Studies in CSR. Business Ethics A European Review, pp. 11-23.

[3] Eemeren, F. H. (1999). Strategic Manoevering in Argumentative Discourse. Discourse Studies, 480-497.

[4] Fugere, B., Hardaway, C., \& Warshawsky, J. (2005). Why Business People Speak Like Idiots: A Bullfighter's Guide. New York City: Simon and Schuster.

[5] Grouse, L. (2016, July 1). Post Hoc Ergo Propter Hoc. Journal of Thoraic Disease, pp. 511-512.

[6] Hanks, C. (2019, February 24). Texas State University. Retrieved from TxStateEdu: https://www.txstate.edu/philosophy/resources/fallacy-definitions/False-Dilemma.html

[7] Kahneman, D. (2011). Thinking, Fast and Slow. New York City: Farrar, Straus and Giroux.

[8] Lode, E. (1999). Slippery Slope Arguments and Legal Reasoning. California Law Review, 1469-1544.

[9] Lovell, S. (2009). Argument Structure: Secrets of The World's Best Debaters. Amazon E Books.

[10] Minto, B. (1978). The Pyramid Principle. Prentice Hall.

[11] Moshman, D. (1995). Reasoning as self-constrained thinking. Human Development 38, 53-64.

[12] Schwarz, B. B., \& Asterhan, C. (2008). Argumentation \& Reasoning. Elsevier Handbook of Education Psychology: New Perspectives on Learning and Psychology.

[13] Vigen, T. (2015). Spurious Correlations. Amazon E Books.

[14] Walton, D. (1999, January 1). Rethinking the Fallacy of Hasty Generalization. Argumentation, pp. 161-182.

[15] Walton, D. (2006). Fundamentals of Critical Argumentation. Cambridge University Press. 